\title{
Black hole detection with the OWL-Airwatch telescope
}

\author{
Sharada Iyer Dutta \\ Department of Physics, State University of New York at Stony Brook, Stony Brook, New York 11794 \\ Mary Hall Reno \\ Department of Physics and Astronomy, University of Iowa, Iowa City, Iowa 52242 \\ Ina Sarcevic \\ Department of Physics, University of Arizona, Tucson, Arizona 85721 \\ and Theory Division, CERN, CH-1211 Geneva 23, Switzerland \\ (Received 22 April 2002; published 5 August 2002)
}

\begin{abstract}
In scenarios with large extra dimensions and $\mathrm{TeV}$ scale gravity, ultrahigh energy neutrinos produce black holes in their interactions with the nucleons. We show that OWL may observe a large number of black hole events and provide valuable information about the fundamental Planck scale and the number of extra dimensions. OWL is especially well suited to observe black hole events produced by neutrinos from the interactions of cosmic rays with the $3 \mathrm{~K}$ background radiation. Depending on the parameters of the scenario of large extra dimensions and on the flux model, as many as 28 events per year are expected for a Planck scale of $3 \mathrm{TeV}$.
\end{abstract}

DOI: $10.1103 /$ PhysRevD.66.033002

PACS number(s): 13.15. $+\mathrm{g}, 04.50 .+\mathrm{h}$

\section{INTRODUCTION}

A recent proposal of lowering the fundamental Planck scale to the $\mathrm{TeV}$ range has provided a new perspective on studying black hole formation in ultrarelativistic collisions [1]. It has been argued that in particle collisions with energies above the Planck scale $M_{D}\left(M_{D} \sim \mathrm{TeV}\right)$, black holes can be produced and their production and decay can be described semiclassically and thermodynamically [2]. In proton-proton collisions at the Large Hadron Collider (LHC) at CERN with a center-of-mass energy of several $\mathrm{TeV}$, for example, the distinctive characteristics of black hole production would be large multiplicity events [3-5]. The event rates depend strongly on the ratio of the minimum mass of the black hole and the Planck scale and to a lesser extent on the number of extra dimensions [4]. Recently, it has also been pointed out that cosmic ray detectors sensitive to neutrino-induced air showers, such as the large Pierre Auger Observatory, could detect black holes produced in neutrino interactions with the atmosphere [5-9], for example, from interactions of the cosmogenic neutrinos produced in the interaction of cosmic rays with the cosmic microwave background. If interactions are not detected, then cosmic ray detectors could provide constraints on the fundamental Planck scale for any number of extra dimensions [5-10].

In this paper, we show that air shower telescopes such as the Orbiting Wide-Angle Light-Collectors Experiment (OWL) [11] and the Extreme Universe Space Observatory (EUSO) [12] have a very good chance of detecting black holes produced in interactions of ultrahigh energy neutrinos from extragalactic and cosmogenic sources and provide valuable information about the fundamental Planck scale and the number of extra dimensions. We investigate whether the OWL air shower telescopes can probe a region of parameter space that is not accessible to LHC and Auger, and compare the reach of OWL with $\mathrm{a} \mathrm{km}^{3}$ underground detector such as ICECUBE [13].
The OWL experiment will involve photodetectors mounted on two satellites orbiting at $640 \mathrm{~km}$ above Earth's surface. There are three possible satellite configurations: a $500 \mathrm{~km}$ stereo view, a $2000 \mathrm{~km}$ stereo view, and two monocular eyes. In the case of the stereo configurations, the satellites are separated by a distance of $500 \mathrm{~km}$ or $2000 \mathrm{~km}$, respectively, such that they monitor a common region of Earth's atmosphere [14]. The $2000 \mathrm{~km}$ stereo configuration can view a larger volume but, comparatively, the events are observed at a further distance from the satellites. However, in the case of the two monocular eyes configuration, the satellites look down at Earth's surface independently, therefore only one satellite will view an event. The EUSO experiment uses the same principles as the OWL experiment, however it is proposed to be a single eye located on the international space station $380 \mathrm{~km}$ above Earth's surface. The geometric reduction in viewing volume, going from two eyes to one, at a lower altitude, results in a reduction in the event rate by a factor of $\sim 0.2$ compared to OWL. We will concentrate on OWL event rates below.

OWL detects ultrahigh energy neutrino interactions via air fluorescence. The large interaction lengths of neutrinos mean that neutrinos initiate horizontal air showers. By setting an angular threshold of column depth $>1500 \mathrm{~g} / \mathrm{cm}^{2}$ (zenith angle greater than $\sim 50^{\circ}$ ) in the atmosphere, neutrino interactions are distinct from the hadronic and electromagnetic showers initiated by cosmic rays [11]. Because the electron in electron neutrino charged current interactions carries a large fraction ( $\sim 80 \%$ of the incident neutrino energy) and initiates an electromagnetic shower, detection of standard model electron neutrino interactions is favored over muon neutrino interactions. So that we can compare black hole event rates with the standard model event rates, we concentrate on event rates initiated by electron neutrinos and antineutrinos. We comment on the multiplicative factors that are relevant when muon and tau neutrinos plus antineutrinos are included. 


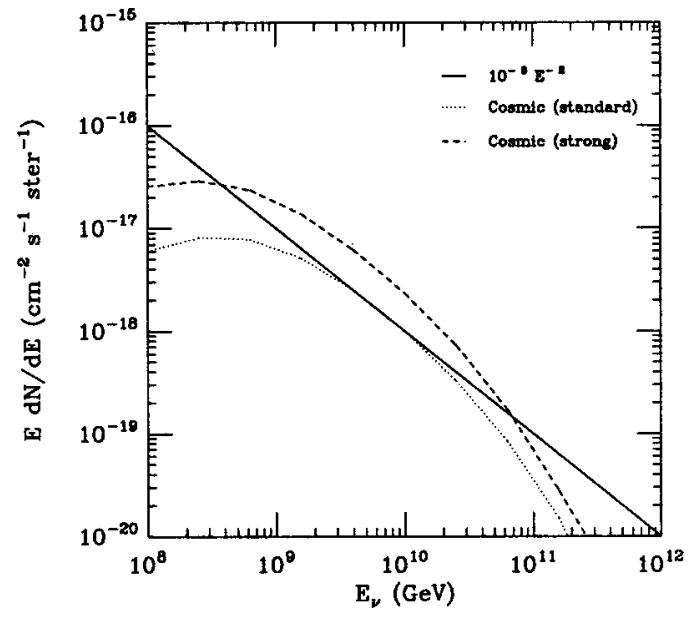

FIG. 1. The cosmogenic electron neutrino plus antineutrino flux evaluated by Engel, Seckel, and Stanev in [18].

Theoretical work has been done to set upper bounds on high energy neutrino fluxes from active galactic nuclei (AGN) jets and gamma ray bursts (GRB) [15]. The bounds are based on the theoretical correlations between the cosmic ray flux and/or the extragalactic gamma ray flux and the neutrino flux. These bounds have some model dependence. An upper bound, depending on cosmic ray source evolution discussed recently by Waxman and Bahcall [15], corresponds to the flux $d N_{\nu} / d E_{\nu}=(1-5) \times 10^{-8}\left(E_{\nu} / \mathrm{GeV}\right)^{-2}$ $\left(\mathrm{GeV} \mathrm{cm} \mathrm{s} \mathrm{sr}^{-1}\right.$ for the sum of muon neutrino plus antineutrino fluxes. The electron neutrino plus antineutrino limits are a factor of 0.5 lower and are dominated by the neutrino component. For convenience, we use

$$
d N_{\nu} / d E_{\nu}=10^{-8}\left(E_{\nu} / \mathrm{GeV}\right)^{-2}\left(\mathrm{GeV} \mathrm{cm}^{2} \mathrm{~s} \mathrm{sr}\right)^{-1}
$$

for the sum of the electron neutrino plus antineutrino bound. In the case of bimaximal $\nu_{\mu} \leftrightarrow \nu_{\tau}$ neutrino oscillations, as favored by the Super-Kamiokande experimental data [16], half of the muon neutrino plus antineutrino flux oscillates into tau neutrinos plus antineutrinos, yielding a ratio of 1:1:1 for electron, muon, and tau neutrino flavors, each of which we take as limited by Eq. (1).

While the sources of ultrahigh energy cosmic rays are not completely understood, cosmic ray fluxes are measured. As the cosmic rays must traverse a portion of the universe filled with the $3 \mathrm{~K}$ background radiation, at sufficiently high energies, neutrinos will be produced by photoproduction of charged pions which decay into neutrinos [17]. We use here a new evaluation of this cosmogenic neutrino flux by Engel, Seckel, and Stanev [18]. They have evaluated the electron neutrino plus antineutrino fluxes (and muon neutrino plus antineutrino fluxes) using photoproduction rates based on the event generator SOPHIA [19]. They have presented results for two models of source evolution, one with a parametrization scaling like $(1+z)^{3}$ for redshift $z<1.9$ (standard evolution), another scaling like $(1+z)^{4}$ for redshift $z<1.9$ (strong evolution). Their results for the sum of the electron neutrino and antineutrino fluxes, for $E_{\nu}>10^{8} \mathrm{GeV}$, are shown in Fig. 1, along with a line associated with the approximate limit of
Eq. (1). The muon neutrino plus antineutrino flux is approximately a factor of 2 larger [18]. The cosmogenic neutrino flux by Engel, Seckel, and Stanev peaks at the same energy $\left[(2-3) \times 10^{8} \mathrm{GeV}\right]$ as the flux calculated by Yoshida and Teshima [20] and by Protheroe and Johnson [21]. The flux of Yoshida and Teshima is slightly narrower, while the agreement with the flux of Protheroe and Johnson is very good.

In the next section we review the black hole cross section. This is followed by a discussion of the evaluation of the OWL event rates and a comparison with the capabilities of ICECUBE. Our conclusions are presented in the final section.

\section{BLACK HOLE CROSS SECTION}

At very high energies and at small impact parameters, in the case of large extra dimensions, neutrino-parton interactions will result in the creation of a black hole. For this to happen, the center-of-mass energy has to be above the Planck scale $M_{D}, \hat{s} \gg M_{D}^{2}$ and the impact parameter has to be much smaller than the Scharzschild radius in $4+n$ dimensions.

In this case, the neutrino-parton cross section is given by

$$
\hat{\sigma}(\nu j \rightarrow \mathrm{BH})=\pi r_{S}^{2}\left(M_{\mathrm{BH}}=\sqrt{\hat{s}}\right) \theta\left(\sqrt{\hat{s}}-M_{\mathrm{BH}}^{\min }\right),
$$

where $r_{S}$ is a Schwarzschild radius given by

$$
r_{S}=\frac{1}{M_{D}}\left[\frac{M_{\mathrm{BH}}}{M_{D}}\left(\frac{2^{n} \pi^{(n-3) / 2} \Gamma\left(\frac{3+n}{2}\right)}{2+n}\right)\right]^{1 /(1+n)} .
$$

Here, $M_{\mathrm{BH}}^{\min } \gg M_{D}$ parametrizes the center-of-mass energy above which the semiclassical reasoning mentioned above is assumed to be valid. It has been argued that in the case of cosmic ray showers initiated by black hole decay, one can relax this constraint because the details of the final state are not that important [22]. In our study, we will vary $M_{\mathrm{BH}}^{\mathrm{min}}$ from $1 \cdot M_{D}$ to $10 \cdot M_{D}$. We will consider $M_{D} \geqslant 1 \mathrm{TeV}$ for $n$ $=2,4,6$.

The neutrino-nucleon cross section for black hole production is given by

$$
\sigma(\nu N \rightarrow \mathrm{BH})=\sum_{i} \int_{\left(M_{\mathrm{BH}}^{\min }\right)^{2} / s}^{1} d x \hat{\sigma}_{i}^{\mathrm{BH}}(x s) f_{i}\left(x, Q^{2}\right),
$$

where $s$ is the center-of-mass energy squared, $s=2 m_{N} E_{\nu}$, and $f_{i}\left(x, Q^{2}\right)$ is the parton distribution function for parton $i$ [23]. All partons contribute, and the antineutrino-nucleon cross section for black hole production is identical to Eq. (4).

Qualitatively, we are interested in cross sections for $M_{D}$ $\sim 1 \mathrm{TeV}$, since one motivation for $\mathrm{TeV}$-scale compactification is the gauge hierarchy problem. Scales much lower than $1 \mathrm{TeV}$ would be manifest in collider experiments by additional contributions from virtual graviton exchange, requiring $M_{D} \gtrsim 1 \mathrm{TeV}$ [22]. Direct graviton emission is already constrained by the CERN $e^{+} e^{-}$collider LEP to $M_{D}$ $>870 \mathrm{GeV}$ for $n=4$ and $M_{D}>610 \mathrm{GeV}$ for $n=6[24,25]$. 


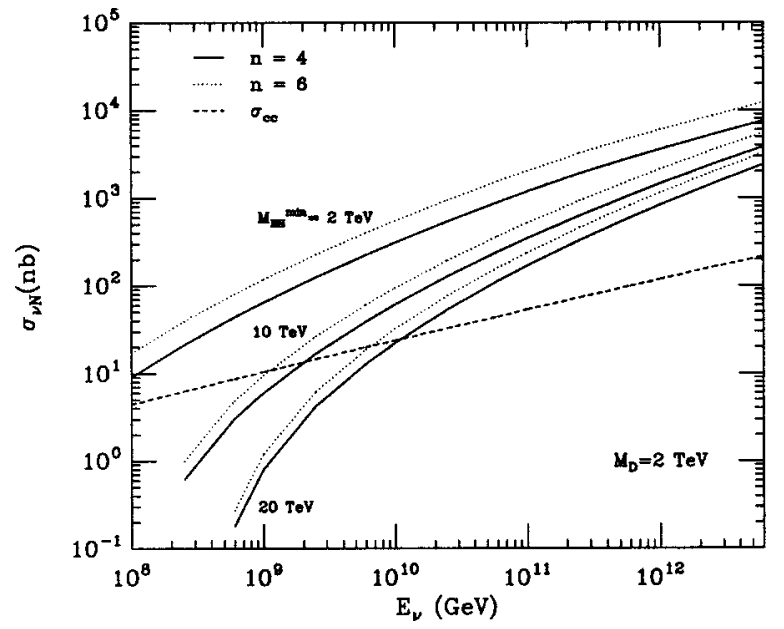

FIG. 2. Cross section for the black hole production in neutrinonucleon scattering as a function of neutrino energy for $n=4,6$ and $M_{D}=2 \mathrm{TeV}$. We also show the standard model charged-current cross section [28].

The radii of $n$ extra dimensions is of the order $R$ $\sim 2 \times 10^{-17} \mathrm{~cm}\left(\mathrm{TeV} / M_{D}\right) \cdot\left(1.2 \times 10^{16} \mathrm{TeV} / M_{D}\right)^{2 / n}$.

Deviations from Newtonian gravity over solar system distances exclude the $n=1$ and $M_{D} \sim 1 \mathrm{TeV}$ possibility [22]. For $n=2$, submillimeter tests of the gravitational inversesquare law constrain $M_{D}>3.5 \mathrm{TeV}$ [26] assuming two equal large extra dimensions. For larger values of $n$, experimental nonobservation of deviations from Newtonian gravity do not constrain $M_{D}>1 \mathrm{TeV}$. There are also astrophysical limits from, for example, supernova cooling [27], however these are more model-dependent.

In Fig. 2, we show the cross sections for black hole production for two values of the number of extra dimensions, $n=4,6$, for $M_{D}=2 \mathrm{TeV}$ as a function of incident neutrino energy for several values of $M_{\mathrm{BH}}^{\mathrm{min}}$. We also show the standard model neutrino cross section [28]. For neutrino energies above $10^{6} \mathrm{GeV}, \sigma(\nu N) \simeq \sigma(\bar{\nu} N)$ to within $5 \%$, so we need not distinguish between incident neutrinos and antineutrinos in our discussion.

For $M_{D}=1 \mathrm{TeV}$, the cross sections are larger than in Fig. 2 , with the range of cross sections of $\sim 10-10^{5} \mathrm{nb}$ for the same range of energies when $M_{\mathrm{BH}}^{\min }=M_{D}$. For $M_{D}=3 \mathrm{TeV}$, at the highest energies, the cross sections in Fig. 2 are reduced by a factor of $\sim 3$. The standard model cross section is exceeded by the black hole production cross section in the energy range of $\sim 10^{8}-10^{11} \mathrm{GeV}$, depending on $n, M_{D}$, and $M_{\mathrm{BH}}^{\min }$.

There has been a suggestion by Voloshin [29] that the geometrical black hole cross section is an overestimate. A suppression factor that depends on the exponential of the black hole entropy has the effect of reducing the geometrical cross section [Eq. (4)] by a factor of $\sim 1 / 3-1 / 8$ for $M_{D}$ $=M_{\mathrm{BH}}^{\min }=1-3 \mathrm{TeV}$ and $n=4,6$ in the relevant OWL energy range: $E_{\nu}=10^{10}-10^{12} \mathrm{GeV}$. For $n=2$, the reduction is more dramatic, as is the case when $M_{\mathrm{BH}}^{\min }$ is large compared to $M_{D}$. This suppression, however, has recently been challenged by several authors [30]. Thus, in this paper we restrict our comments to the geometrical cross section.

From Fig. 2, we see that for the energies of interest for the OWL detector, the largest black hole production cross sections are on the order of $10^{4} \mathrm{nb}$. While these are larger than the standard model neutrino-nucleon cross sections, they are still small compared to typical strong interaction cross sections in the range of tens of millibarns. As a result, even with the enhanced cross sections for black hole production, the neutrino still penetrates deep into the atmosphere, as demonstrated below.

\section{DETECTION OF BLACK HOLE PRODUCTION WITH OWL}

The detection of neutrino production of black holes by interactions of neutrinos with nuclei in the atmosphere follows the same principle as detection of neutrinos via their standard model interactions. Since neutrinos are weakly interacting, they are more likely to penetrate the atmosphere in the horizontal direction, whereas cosmic rays interact in a shell about $20 \mathrm{~km}$ above the surface of the Earth.

To estimate the critical cross section, below which the probability of interaction in the atmosphere is peaked at sea level, one can compare the column depth of the atmosphere with the interaction cross section. The column depth at zenith angle $\theta$ is

$$
X=\int_{0}^{\infty} d x \rho(h(x, \theta))
$$

as measured along the particle trajectory from a point on the surface of the Earth (of radius $R_{\oplus}$ ), in terms of the atmospheric density $\rho$ as a function of altitude $h$ $=\sqrt{R_{\oplus}^{2}+2 x R_{\oplus} \cos \theta+x^{2}}-R_{\oplus}$. To a good approximation, the U.S. Standard Atmosphere (1976) [31] is

$$
\rho_{\text {atm }}(h)=\left\{\begin{array}{c}
1.225 \times 10^{-3} \quad \mathrm{~g} / \mathrm{cm}^{3} \exp (-h / 9.192 \mathrm{~km}), \\
h<10 \mathrm{~km} \\
1.944 \times 10^{-3} \mathrm{~g} / \mathrm{cm}^{3} \exp (-h / 6.452 \mathrm{~km}), \\
h \geqslant 10 \mathrm{~km} .
\end{array}\right.
$$

Numerically, the column depth for neutrinos arriving vertically is $10^{3}$ cmwe, while the column depth for neutrinos arriving horizontally is $3.6 \times 10^{4} \mathrm{cmwe}$. By comparison, the neutrino interaction length is

$$
\lambda_{\nu}=1.7 \times 10^{9} \cdot(\sigma / \mathrm{nb})^{-1} \text { cmwe, }
$$

so for $\sigma<\sigma_{\text {crit }} \simeq 5 \times 10^{4} \mathrm{nb}$, the horizontal column depth is larger than the interaction length. The black hole cross section in Fig. 2 is below $10^{4} \mathrm{nb}$ for $E_{\nu}<10^{12} \mathrm{GeV}$, so $X / \lambda_{\nu}$ $<0.2$ for horizontal neutrinos. In fact, the cosmogenic neutrino flux falls with energy more rapidly than $E_{v}^{-2}$ above $E_{\nu} \sim 10^{8} \mathrm{GeV}$, and the Waxman-Bahcall bound falls with $E_{\nu}^{-2}$, so the bulk of the contribution to the event rate is at $E_{\nu} \ll 10^{12} \mathrm{GeV}$ where $X / \lambda_{\nu} \ll 1$.

The standard model electron neutrino effective aperture is shown in Fig. 3 [11]. At high energies, the effective aperture 


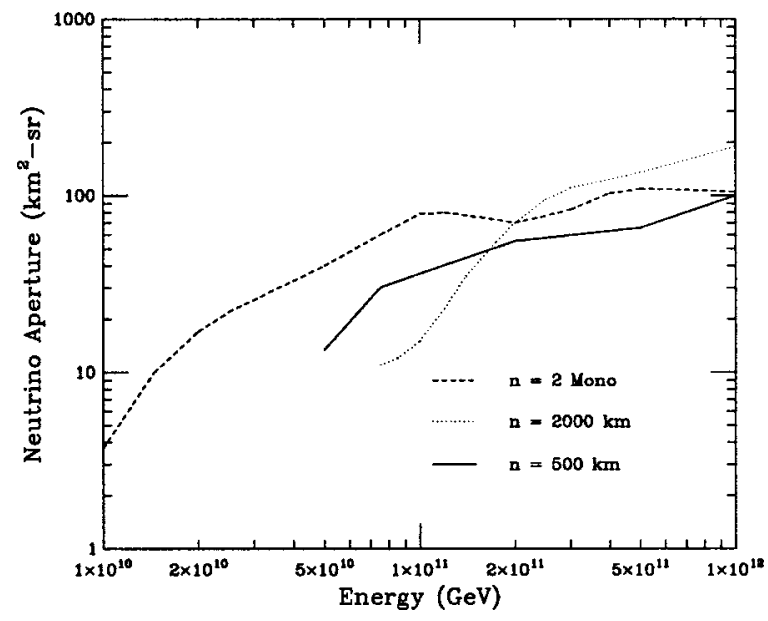

FIG. 3. OWL electron neutrino (standard model) effective aperture as a function of energy for $500 \mathrm{~km}, 2000 \mathrm{~km}$ satellite separation and 2 monocular eyes [11].

roughly scales with energy as the standard model neutrinonucleon cross section does. To a good approximation, all of the energy of the decaying black hole is deposited into hadronic or electromagnetic showers, just as all of the energy in electron neutrino charged current interactions is deposited in the shower. Since the neutrino interaction length, even when black hole production is included, is small, one may evaluate the number of black holes detected with OWL by rescaling the neutrino aperture for electron neutrinos by the ratio of the black hole cross section to the neutrino-nucleon standard model charged current cross section $\left(\sigma_{\mathrm{CC}}^{\mathrm{SM}}\right)$ :

$$
N=T \int_{E_{\min }}^{\infty} \epsilon A_{\mathrm{Nuc}}(E) \frac{\sigma_{\mathrm{BH}}\left(E_{\nu}\right)}{\sigma_{\mathrm{CC}}^{\mathrm{SM}}\left(E_{\nu}\right)} \frac{d N_{\nu}}{d E_{\nu}} d E_{\nu}
$$

where $T$ is the duration of data taking, $\epsilon=0.1$ is the duty cycle, $A_{\text {Nuc }}(E)$ is the OWL aperture as it appears in Fig. 3 [11], $d N_{\nu} / d E_{\nu}$ is the neutrino flux, and $\sigma_{\mathrm{BH}}\left(E_{\nu}\right)$ is the cross section for the production of black hole.

The resulting event rates are shown in Figs. 4 and 5 for the standard evolution and the strong evolution $\nu_{e}+\bar{\nu}_{e}$ cosmogenic fluxes of Engel, Seckel, and Stanev [18]. We show the results for the two monocular eyes configuration. The stereo configurations have larger threshold energies which gives smaller event rates. The standard model contributions are also shown. In the case of the cosmogenic electron neutrino plus antineutrino flux obtained with the standard evolution, the background from the standard model charged current interaction is very small (about 0.4 events per year), while the showers from black hole evaporation give two events per year for $M_{D}=M_{\mathrm{BH}}^{\min }=3 \mathrm{TeV}$ and $n=4$, and give between three and eight events for $M_{D}=2 \mathrm{TeV}, M_{\mathrm{BH}}^{\min }$ $\leqslant 4 M_{D}$, and $n=4$. As many as 100 events are possible with $M_{D}=1 \mathrm{TeV}$ and $n=6$. The cosmogenic neutrino flux with strong evolution gives larger event rates, by about a factor of 2 , both for the signal and the background.

Similar results for event rates are obtained with the Waxman-Bahcall bound on the neutrino flux from optically

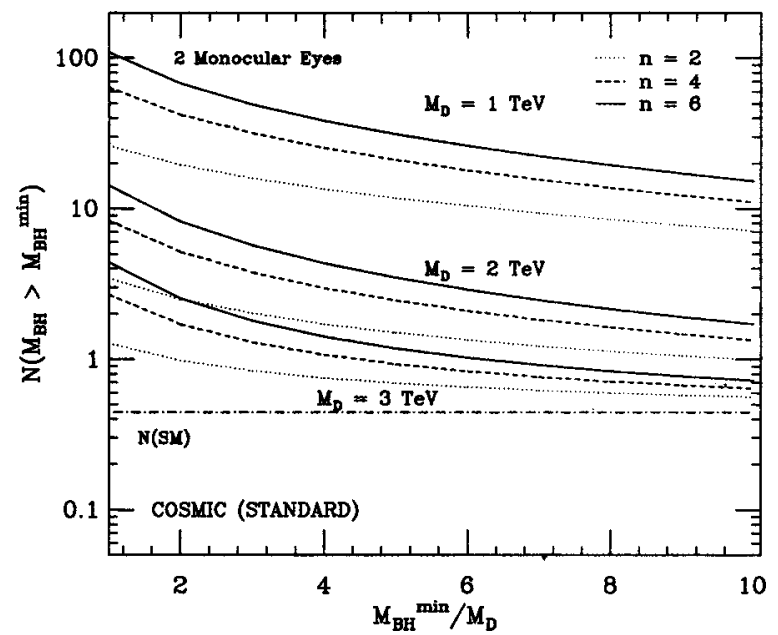

FIG. 4. Event rate per year for black hole production plus standard model background as a function of $M_{\mathrm{BH}}^{\min } / M_{D}$ for cosmic (standard) incident $\nu_{e}+\bar{\nu}_{e}$ flux. The OWL rates are shown in the case of 2 monocular eyes for $n=2,4,6$ extra dimensions and $M_{D}$ $=1,2,3 \mathrm{TeV}$. We also show separately the standard model event rates.

thin sources as represented by Eq. (1). These are shown for the two monocular eyes in Fig. 6. For $M_{D}=1 \mathrm{TeV}$ and $n$ $\geqslant 4$, we find that OWL would detect between 20 and 200 events per year, about two orders of magnitude above the standard model predictions. For larger $M_{D}$, the rates decrease so that for $M_{D}=M_{\mathrm{BH}}^{\min }=2 \mathrm{TeV}$ and $n \geqslant 4$, there are tens of events per year. For $M_{D}=M_{\mathrm{BH}}^{\min }=3 \mathrm{TeV}$ and $n \geqslant 4$ there are a handful of events with a background of 0.8 events.

The event rates above can be compared with contained rates for electron neutrino interaction in a $\mathrm{km}^{3}$ detector like ICECUBE. We find a handful of event in ICECUBE using an

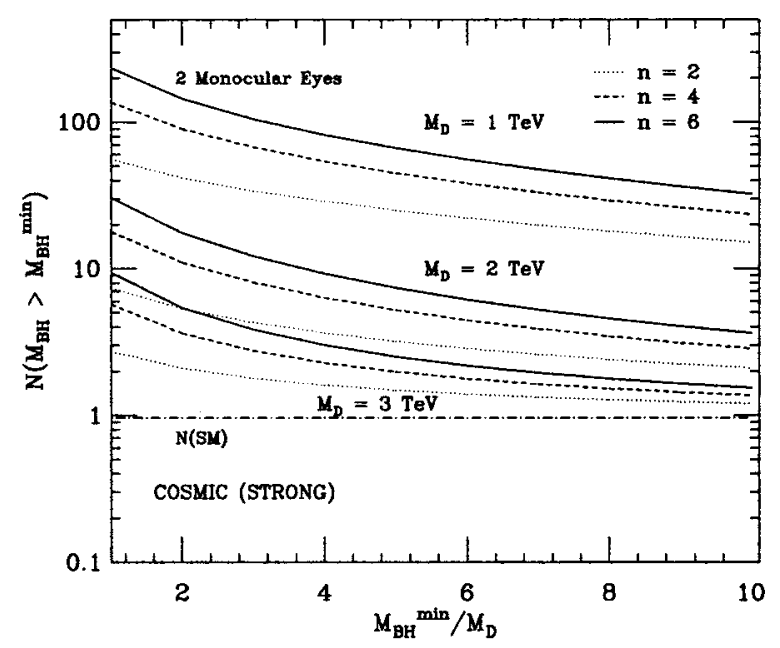

FIG. 5. Event rate per year for black hole production plus standard model background as a function of $M_{\mathrm{BH}}^{\mathrm{min}} / M_{D}$ for cosmic (strong) incident $\nu_{e}+\bar{\nu}_{e}$ flux. The OWL rates are shown in the case of 2 monocular eyes for $n=2,4,6$ extra dimensions and $M_{D}$ $=1,2,3 \mathrm{TeV}$. We also show separately the standard model event rates. 


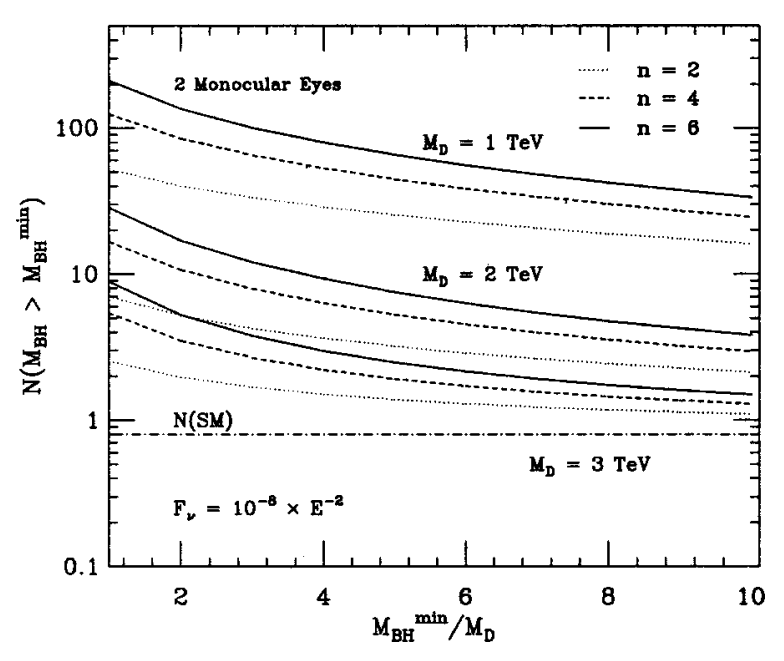

FIG. 6. Event rate per year for black hole production plus standard model background as a function of $M_{\mathrm{BH}}^{\mathrm{min}} / M_{D}$ for $d N_{\nu} / d E_{\nu}$ $=10^{-8} E_{\nu}^{-2}$ incident neutrino flux. The OWL rates are shown in the case of 2 monocular eyes for $n=2,4,6$ extra dimensions and $M_{D}$ $=1,2,3 \mathrm{TeV}$. We also show separately the standard model event rates.

energy threshold of $10^{8} \mathrm{GeV}$ for $M_{D}=1,2,3 \mathrm{TeV}$, depending on the value of the number of extra dimensions, for all three fluxes. The standard model rates per year are $0.08(0.03$, 0.09 ) for the Waxman-Bahcall bounded $E^{-2}$ (standard cosmogenic, strong cosmogenic) flux with the same energy threshold. Even with the most optimistic parameters, $n=6$ and $M_{\mathrm{BH}}^{\mathrm{min}}=M_{D}=1 \mathrm{TeV}$, the Waxman-Bahcall bounded $E^{-2}$ flux (standard cosmogenic, strong cosmogenic) annual event rates for downward contained events in ICECUBE are 8.0 $(3.9,10.5)$. OWL rates for the same parameters are on the order of 100 events per year. Our ICECUBE rates agree qualitatively with the recent results of Alvarez-Muniz et al. [32], who used a lower threshold energy, included $\nu_{\mu}$ and $\nu_{\tau}$ fluxes, and had different assumptions about the shower energy in the decay of the black hole. We comment on the consequences of these different assumptions for the OWL rates in the next sections.

The OWL rates as shown in the figures are a factor of more than 20 times higher than ICECUBE downward contained event rates with an energy threshold of $10^{8} \mathrm{GeV}$. Lowering the energy threshold for ICECUBE increases the event rates for the Waxman-Bahcall flux bound, but decreases the signal to background ratio. The cosmogenic neutrino flux is less sensitive to the energy threshold because it does not fall $\sim E_{\nu}^{-2}$ for $E_{\nu}<10^{8} \mathrm{GeV}$. ICECUBE should be able to detect black hole events (showers and muons) with $E_{\mathrm{th}} \sim 10^{5} \mathrm{GeV}$ if $M_{D} \leqslant 2 \mathrm{TeV}$ and $n \geqslant 6$ [32], however at higher energies OWL will have more sensitivity.

\section{DISCUSSION}

The event rates in Figs. 4-6 include only incident electron neutrino and antineutrino fluxes. Since the cross section for neutrino production of black holes is lepton flavor-blind, the black hole production rates can be multiplied by the ratio of the total neutrino plus antineutrino flux to the electron neutrino plus antineutrino flux. The net effect is essentially a factor of 3 , since generically, the neutrinos are coming from $\pi^{+} \rightarrow \nu_{\mu} \mu^{+} \rightarrow \nu_{\mu} \bar{\nu}_{\mu} \nu_{e} e^{+}$. This is borne out numerically in, for example, the cosmogenic flux of Engel, Seckel, and Stanev. This leads to a factor of 3 enhancement of all of the black hole rates in Figs. 4-6. The standard model rates are not necessarily increased by such a large factor because the charged lepton carries, on average, about $80 \%$ of the incident neutrino energy after the charged current interaction, leaving the shower with $20 \%$ of the incident neutrino energy. For a flux falling like $E_{\nu}^{-2}$ and standard model cross sections that increase like $\sim E_{\nu}^{0.4}[28]$, the rate of hadronic showers from the charged current interaction will be suppressed by a factor of $\sim(0.2)^{0.6}=0.4$. By including all three neutrino flavors, the standard model rates increase by roughly a factor of 2 . For the contained rates induced by cosmogenic neutrinos with a minimum shower energy around $10^{8} \mathrm{GeV}$, the standard model rates are increased by a factor of $\sim 3$ for the total neutrino flux. This is due to the relative insensitivity of the event rate to the minimum shower energy with the flatter shape of the cosmogenic $E_{\nu} d N_{\nu} / d E_{\nu}$ below $E_{\nu} \sim 10^{9} \mathrm{GeV}$.

A related issue is the uncertainty in the event rates presented here due to the approximation that all of the black hole energy goes into the showers. It has been argued that only $\sim 75 \%$ of the incident neutrino energy ends up in the hadronic shower associated with the black hole decay [32]. Because the black hole cross sections grow with incident neutrino energy like $\sim E_{\nu}^{0.5}-E_{\nu}^{0.8}$, given $d N_{\nu} / d E_{\nu} \sim E_{\nu}^{-2}$ and $E_{\text {shr }}=0.75 E_{\nu}$, the rates will be suppressed by $(0.75)^{0.2}$ $-(0.75)^{0.5}$, between a $5-15 \%$ decrease in the rates. The suppression will be even less for the contained cosmogenic rates since the shower threshold dependence is weak for $E_{\text {shr }}^{\min } \sim 10^{8} \mathrm{GeV}$.

Neutrino fluxes that consistently decrease like $E_{\nu}^{-2}$ will most likely be seen at lower energy thresholds than considered here, in detectors like ICECUBE. Alvarez-Muñiz et al. in Ref. [32] have emphasized the different (and complementary) signals of muons, taus, and showers that can be used as diagnostics. For the cosmogenic flux, the spectrum is such that higher energies are emphasized and larger volumes are required.

The OWL telescope has the capability of probing the cosmogenic flux farther than ground-based air shower experiments. The nonobservation of an excess of shower events at the AGASA air shower array led to limits on the black hole production parameters and require $M_{D} \geqslant 1.3-1.8 \mathrm{TeV}$ [22]. The OWL standard model rates are at the 1.5-3 events per year level, depending on the flux, when one includes all flavors of neutrinos. The rates for OWL are ten to hundreds of events per year for $M_{D}=1 \mathrm{TeV}$ for $M_{\mathrm{BH}}^{\min }=1-10$, even in the case of the conservative evolution of the cosmogenic flux. Similar results are found for the Waxman-Bahcall flux, which represents the upper bound for optically thin sources. Multiplying the black hole rates for cosmogenic fluxes in the figures by a factor of 3 means that for $M_{\mathrm{BH}}^{\mathrm{min}}=5(1) M_{D}, M_{D}$ $=3 \mathrm{TeV}, n=6$, the annual signal event rates will be on the 
order of 4.4 (24) for the strong evolution cosmogenic flux model and 2.4 (12) for the standard evolution. This is a much larger reach in parameter space than the terrestrial experiments. One year of data taking would be sufficient for OWL to have a unique opportunity to detect black holes, or to probe the fundamental Planck scale up to $M_{D}$ $=3 \mathrm{TeV}$ for $n \geqslant 4$. OWL is set for possible implementation after 2007. EUSO is proposed to go on the International Space Station in 2006. EUSO, with a projected event rate on the order of $1 / 5$ of the OWL rate, will be able to probe regions of parameter space intermediate between ICECUBE and OWL.

\section{ACKNOWLEDGMENTS}

The work of S.I.D. has been supported in part by National Science Foundation Grant No. 0070998. The work of I.S. has been supported in part by the DOE under Contracts No. DEFG02-95ER40906 and No. DE-FG03-93ER40792. The work of M.H.R. has been supported in part by National Science Foundation Grant No. PHY-9802403. We thank J. Krizmanic for discussions and providing the neutrino acceptance for OWL. We thank T. Stanev for providing data files with the ESS fluxes. I.S. thanks the theory group at CERN for its hospitality.
[1] I. Antoniadis, Phys. Lett. B 246, 377 (1990); N. ArkaniHamed, S. Dimopoulos, and G.R. Dvali, ibid. 429, 263 (1998); I. Antoniadis, N. Arkani-Hamed, S. Dimopoulos, and G.R. Dvali, ibid. 436, 257 (1998).

[2] R. Emparan, G.T. Horowitz, and R.C. Myers, Phys. Rev. Lett. 85, 499 (2000).

[3] S.B. Giddings and S. Thomas, Phys. Rev. D 65, 056010 (2002); S. Dimopoulos and G. Landsberg, Phys. Rev. Lett. 87, 161602 (2001).

[4] T.G. Rizzo, in Proc. of the APS/DPF/DPB Summer Study on the Future of Particle Physics (Snowmass, 2001), edited by R. Davidson and C. Quigg, hep-ph/0111230.

[5] A. Ringwald and H. Tu, Phys. Lett. B 525, 135 (2002).

[6] R. Emparan, M. Masip, and R. Rattazzi, Phys. Rev. D 65, 064023 (2002).

[7] J.L. Feng and A.D. Shapere, Phys. Rev. Lett. 88, 021303 (2002).

[8] L. Anchordoqui and H. Goldberg, Phys. Rev. D 65, 047502 (2002).

[9] Y. Uehara, Prog. Theor. Phys. 107, 621 (2002).

[10] C. Tyler, A.V. Olinto, and G. Sigl, Phys. Rev. D 63, 055001 (2001).

[11] OWL-AirWatch Collaboration, J.F. Krizmanic et al., in Proceedings of the 26th International Cosmic Ray Conference (ICRC 99), Salt Lake City, 1999, Cosmic Ray, Vol. 2, pp. 388-391; J.F. Krizmanic (private communications); http:// lheawww.gsfc.nasa.gov/docs/gamcosray/ hecr/owl_new.html

[12] O. Catalano, Nuovo Cimento Soc. Ital. Fis., C 24C, 445 (2001)

[13] AMANDA Collaboration, F. Halzen et al., in 26th International Cosmic Ray Conference (ICRC 99), Salt Lake City, 1999, edited by B.L. Dingus, D.B. Kieda, M.H. Salamon (AIP, Melville, NY, 2000), pp. 428-431.

[14] J.F. Krizmanic and The OWL Collaboration, Proceedings of the ICRC 2001: 861 (2001).

[15] E. Waxman and J.N. Bahcall, Phys. Rev. D 59, 023002 (1999); J.N. Bahcall and E. Waxman, ibid. 64, 023002 (2001); K. Mannheim, R.J. Protheroe, and J.P. Rachen, ibid. 63, 023003 (2001).

[16] Super-Kamiokande Collaboration, Y. Fukuda et al., Phys. Rev. Lett. 81, 1562 (1998).
[17] K. Greisen, Phys. Rev. Lett. 16, 748 (1966); G.T. Zatsepin and V.A. Kuzmin, Pis'ma Zh. Éksp. Teor. Fiz. 4, 114 (1966) [JETP Lett. 4, 78 (1966)]; V.S. Beresinsky and G.T. Zatsepin, Phys. Lett. 28B, 423 (1969).

[18] R. Engel, D. Seckel, and T. Stanev, Phys. Rev. D 64, 093010 (2001).

[19] A. Mücke et al., Comput. Phys. Commun. 124, 290 (2000).

[20] S. Yoshida and M. Teshima, Prog. Theor. Phys. 89, 833 (1993).

[21] R.J. Protheroe and P.A. Johnson, Astropart. Phys. 4, 253 (1996); 5, 215(E) (1996).

[22] L.A. Anchordoqui, J.L. Feng, H. Goldberg, and A.D. Shapere, Phys. Rev. D 65, 124027 (2002).

[23] H.L. Lai et al., Phys. Rev. D 55, 1280 (1997).

[24] L3 Collaboration, M. Acciarri et al., Phys. Lett. B 464, 135 (1999).

[25] L3 Collaboration, M. Acciarri et al., Phys. Lett. B 470, 281 (1999).

[26] C.D. Hoyle, U. Schmidt, B.R. Heckel, E.G. Adelberger, J.H. Gundlach, D.J. Kapner, and H.E. Swanson, Phys. Rev. Lett. 86, 1418 (2001).

[27] S. Cullen and M. Perelstein, Phys. Rev. Lett. 83, 268 (1999); V.D. Barger, T. Han, C. Kao, and R.J. Zhang, Phys. Lett. B 461, 34 (1999); L.J. Hall and D.R. Smith, Phys. Rev. D 60, 085008 (1999); C. Hanhart, J.A. Pons, D.R. Phillips, and S. Reddy, Phys. Lett. B 509, 1 (2001); S. Hannestad and G. Raffelt, Phys. Rev. Lett. 87, 051301 (2001); 88, 071301 (2002).

[28] R. Gandhi, C. Quigg, M.H. Reno, and I. Sarcevic, Phys. Rev. D 58, 093009 (1998); Astropart. Phys. 5, 81 (1996).

[29] M.B. Voloshin, Phys. Lett. B 518, 137 (2001); 524, 376 (2002).

[30] D.M. Eardley and S.B. Giddings, Phys. Rev. D (to be published), gr-qc/0201034; S.D. Hsu, hep-ph/0203154; A. Jevicki and J. Thaler, Phys. Rev. D (to be published), hep-th/0203172.

[31] See, for example, the CRC Handbook of Chemistry and Physics, 75th ed., edited by David R. Lide (CRC Press, Boca Raton, FL, 1994), p. 14. The parametrization in Eq. (6) appears in Ref. [14].

[32] J. Alvarez-Muñiz, J.L. Feng, F. Halzen, T. Han, and D. Hooper, Phys. Rev. D 65, 124015 (2002); M. Kowalski, A. Ringwald, and H. Tu, Phys. Lett. B 529, 1 (2002). 